\title{
Piezoelectric Generator Harvesting Bike Vibrations Energy to Supply Portable Devices
}

\author{
E. Minazara ${ }^{1}$, D. Vasic ${ }^{1,2}$ and F. Costa ${ }^{1,3}$ \\ ${ }^{1}$ SATIE (CNRS UMR 8029), PRES UNIVERSUD, ENS Cachan, 61 av. du Président Wilson 94230 Cachan France \\ ${ }^{2}$ Université de Cergy-Pontoise 95031 \\ ${ }^{3}$ IUFM Paris 12
}

Phone: +0033 1474076 37/Fax number:+0033 1474021 99, e-mail: minazara@ satie.ens-cachan.fr, costa@ satie.enscachan.fr, vasic@satie.ens-cachan.fr

\begin{abstract}
.
With the decrease in energy consumption of portable electronic devices, the concept of harvesting renewable energy in human surrounding arouses a renewed interest. In this context, we have developed a piezoelectric generator that harvests mechanical vibrations energy available on a bicycle. Embarked piezoelectric transducer, which is an electromechanical converter, undergoes mechanical vibrations therefore produce electricity. A static converter transforms the electrical energy in a suitable form to the targeted portable application. Values of generated electrical power are reported and commented.
\end{abstract}

\section{Key words}

Bicycle mechanical vibrations, energy harvesting, piezoelectric material, uninterrupted power supplies, portable device.

\section{Introduction}

Electrical devices on a bicycle, either front or rear lamp for lighting at night, or digital displays such as speedometer, are fed almost exclusively via a dynamo, or by batteries. Both power systems have disadvantages. With the dynamo, cyclist is obliged to provide more effort than necessary for the movement, since it takes an extra effort to compensate for the friction of the dynamo. The use of battery can be restrictive and not eco-friendly. In this study, which is a first in the literature, we propose an alternative solution to the dynamo and an improvement for the battery lifetime. We have developed a piezoelectric generator, which is a clean and durable solution. Piezoelectric generators employ active materials that generate a charge when mechanically activated. Today we see more and more applications using piezoelectric transducers. Their use as a source of electrical energy presents increasing interest for embarked electronic devices, low power consumption (less than 1 Watt) such as lamps based LED (LightEmitting Diode), displays or sensors [1,2]. Noticing that a bicycle in movement is vibrating permanently, and that these vibrations are vectors of mechanical energy, we can recover and convert the mechanical energy contained in these vibrations into electrical energy by using electromechanical transducers [1,2,3], such as piezoelectric materials. The electrical energy thus produced can be used to power devices aboard the bike, or other portable devices that the cyclist uses.

\section{Fundamentals of piezoelectric material}

The conversion of mechanical energy into electrical one is generally achieved by converters alternator type or commonly known dynamo. But there are other physical phenomena including piezoelectricity that can also convert mechanical movements into electricity.

The piezoelectric effect exists in two domains, the first is the direct piezoelectric effect that describes the material's ability to transform mechanical strain into electrical charge, the second form is the converse effect, which is the ability to convert an applied electrical potential into mechanical strain energy figure 1 .

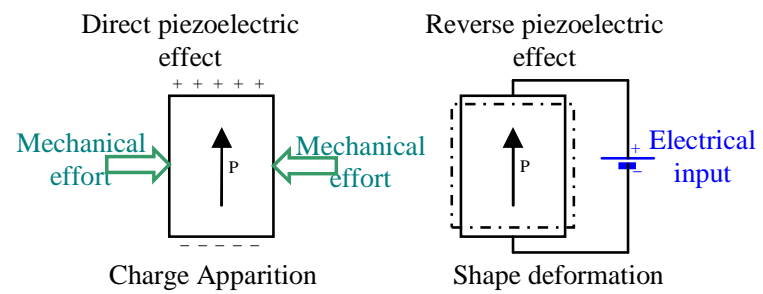

Fig. 1. Electromechanical conversion via piezoelectricity phenomenon

The direct piezoelectric effect is responsible for the materials ability to function as a sensor and the converse piezoelectric effect is accountable for its ability to function as an actuator. A material is deemed piezoelectric when it has this ability to transform electrical energy into mechanical strain energy, and the 
likewise transform mechanical strain energy into electrical charge.

The piezoelectric materials that exist naturally as quartz were not interesting properties for the production of electricity, however artificial piezoelectric materials such as PZT (Lead Zirconate Titanate) present advantageous characteristics.

Piezoelectric materials belong to a larger class of materials called ferroelectrics. One of the defining traits of a ferroelectric material is that the molecular structure is oriented such that the material exhibits a local charge separation, known as an electric dipole. Throughout the artificial piezoelectric material composition the electric dipoles are orientated randomly, but when a very strong electric field is applied, the electric dipoles reorient themselves relative to the electric field; this process is termed poling. Once the electric field is extinguished, the dipoles maintain their orientation and the material is then said to be poled. After the poling process is completed, the material will exhibit the piezoelectric effect. The mechanical and electrical behaviour of a piezoelectric material can be modelled by two linearized constitutive equations. These equations contain two mechanical and two electrical variables. The direct effect and the converse effect may be modelled by the following matrix equations:

\section{Direct Piezoelectric Effect: Converse Piezoelectric Effect:$$
\mathrm{D}=d . \mathrm{T}+\varepsilon^{T} \cdot E(1)
$$$$
\mathrm{S}=s^{E} \cdot \mathrm{T}+d_{\mathrm{t}} \cdot E(2)
$$

Where $\mathrm{D}$ is the electric displacement vector, $\mathrm{T}$ is the stress vector, $\varepsilon^{T}$ is the dielectric permittivity matrix at constant mechanical stress, $\mathrm{s}^{\mathrm{E}}$ is the matrix of compliance coefficients at constant electric field strength, $\mathrm{S}$ is the strain vector, $d$ is the piezoelectric constant matrix, and $\mathrm{E}$ is the electric field vector. The subscript $t$ stands for transposition of a matrix.

When the material is deformed or stressed an electric voltage can be recovered along any surface of the material (via electrodes). Therefore, the piezoelectric properties must contain a sign convention to facilitate this ability to recover electric potential in three directions. For the sake of keeping this discussion simple, the piezoelectric material can be generalized for two cases. The first is the stack configuration that operates in the 33 mode and the second is the bender, which operates in the 13 mode. The sign convention assumes that the poling direction is always in the " 3 " direction; with this point the two modes of operation can be understood figure 2 .

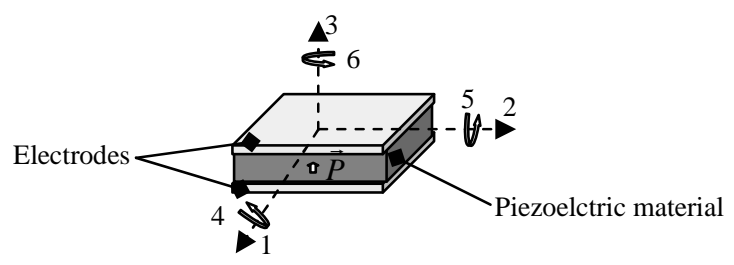

Fig. 2. Defining the lines of a piezoelectric material

In the -33 mode (Figure 3a), the electric voltage is recovered in the " 3 " direction and the material is strained in the poling or " 3 direction, in the -31 mode (Figure $3 b$ ), the electric voltage is measured in the " 3 " direction and the material is strained in the "1" direction or perpendicular to the poling direction. These two modes of operation are particularly important when defining the electromechanical coupling coefficient such as d. Thus $d_{13}$ refers to the sensing coefficient for a bending element poled in the " 3 " direction and strained along " 1 ".

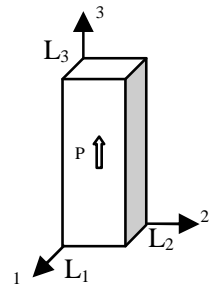

Fig.3a. 33 mode

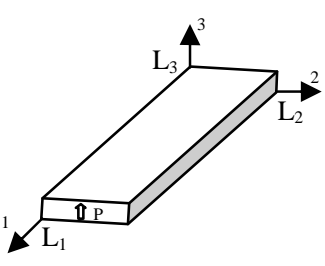

Fig.3b. 31 mode
The coefficients $d_{33}$ of a piezoelectric bar, $(C / N)$ shown in Table I, link the amount of electrical charge (Coulomb), appearing on an electrode perpendicular to the axis 3, to the strain (Newton) applied on both ends.

TABLE I. - Typical piezoelectric materials coefficients

\begin{tabular}{|l|c|}
\hline Material & $\mathrm{d}_{33}\left(10^{-12} \mathrm{C} / \mathrm{N}\right)$ \\
\hline Quartz & 2.3 \\
\hline $\mathrm{BaTiO}_{3}$ & 90 \\
\hline $\mathrm{PbTiO}_{3}$ & 120 \\
\hline PZT & 560 \\
\hline PZN-9PT & 2500 \\
\hline
\end{tabular}

For the PZT, $\mathrm{d}_{33}=560(10-12 \mathrm{C} / \mathrm{N})$ means that $1 \mathrm{~N}$ applied strain produces 560 10-12 C electrical charge.

\section{Piezoelectric generator principle}

The vibrations energy harvesting principle using piezoelectric materials [4] is illustrated in figure 4. The conversion chain starts with a mechanical energy source: bike. Bike vibrations are converted into electicity via piezoelectric element. The electricity produced is thereafter formated by a static converter before supplying a storage system or the load (electrical device).

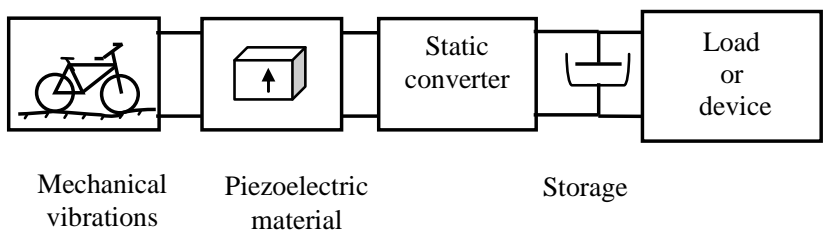

Fig. 4. General diagram of generator based vibrations energy harvesting using piezoelectric material

In this study, before developing bike piezoelectric generator, it was essential to begin with a mechanical vibrations sources identification that means carrying out vibrations accelerations and frequencies measurement and analysis. So we have carried out measurement at different locations of an experimental bicycle (Figure 5) to identify the place where harvesting more energy is possible. We could then develope a piezoelectric generator adapted to the identified natural mode of vibration of the bicycle. 


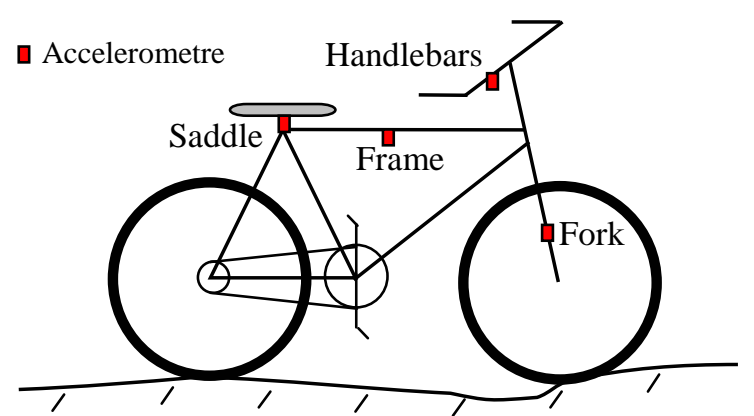

Fig. 5. Accelerometers places

\section{Bike Mechanical Vibrations}

We have equipped the bike with four accelerometers (Figure 5) and a mobile vibrometer "SVAN 948" which includes 4 channels of data acquisition. It is then possible to carry out four simultaneous measurements of vibrations acceleration respectively at the fork, the handlebars, the saddle, and the frame. The accelerometers are mounted vertically to measure only the vertical component of acceleration.

Figure 6 shows the four simultaneous vibrations spectra, respectively, at the fork, handlebars, seat and the frame.

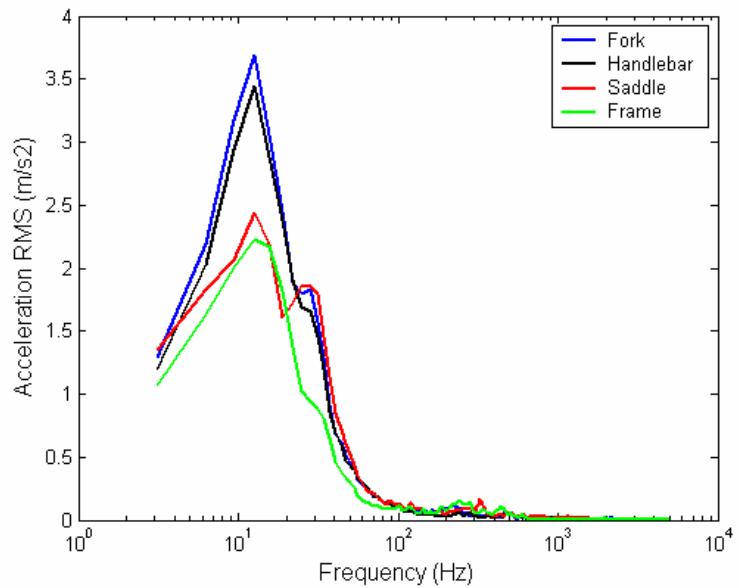

Fig. 6. Vibrations acceleration measurement for the case of a bicycle rolling on uneven path at $8 \mathrm{~km} / \mathrm{h}$ speed

The influence of different runway profiles (Figure 7), cycle track, worn trail, paved runway, as well as the influence of the speed of bicycle on the vibrations acceleration has been investigated. The bicycle speed has been measured through an electronic tachometer.

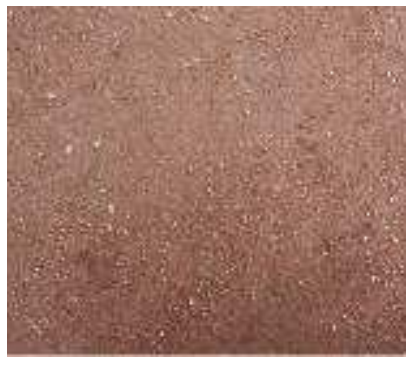

Clay track

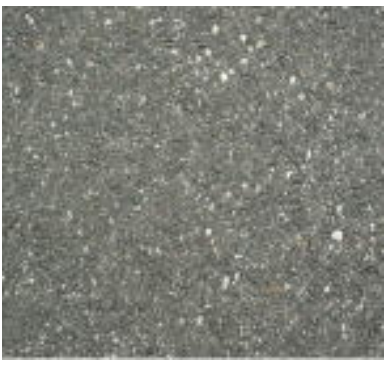

Cycle track

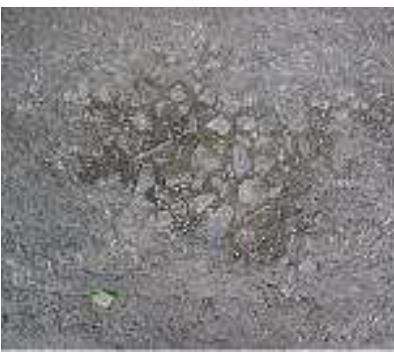

Worn trail

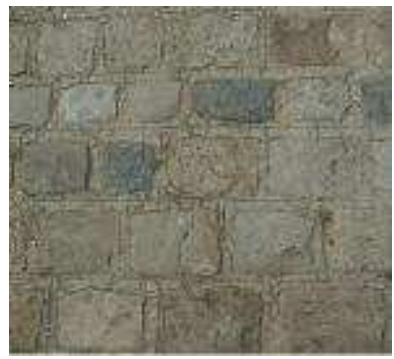

Paved runway
Fig. 7. Track profiles tested.

We noticed that it is at the handlebar or at the fork where the vibration is maximal (see Figure 6). The vibration acceleration is even more important when the displacement speed of the bicycle increases.

The first peak (Figure 6 and 8) corresponds to the natural frequency of bike + cyclist. This peak is observed around $12.5 \mathrm{~Hz}$, regardless of the speed and the track. The interesting frequency band to harvest energy, where $80 \%$ of the energy of the vibrations is concentrated, is approximately between $10 \mathrm{~Hz}$ and $30 \mathrm{~Hz}$ (see Figures 8 and 9).

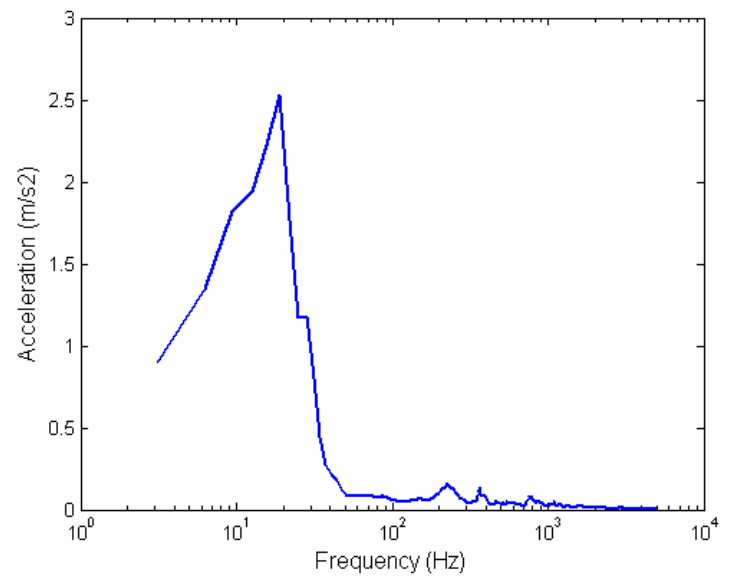

Fig. 8. Vibrations spectrum on the fork, cycle track, $21 \mathrm{~km} / \mathrm{h}$ speed.

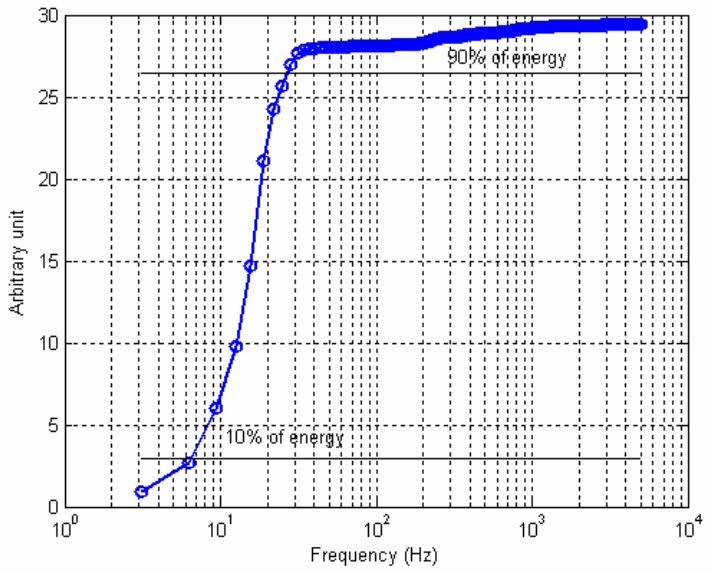

Fig. 9. Cumulative sum of the acceleration squared

It is therefore essential to design a piezoelectric generator that resonates in the frequency range of $10 \mathrm{~Hz}$ to $30 \mathrm{~Hz}$, where $80 \%$ of energy vibration is available. 


\section{Beam-type piezoelectric transducer}

The measured vibration levels remain relatively low since $10 \mathrm{~ms}^{-2}$ is reached almost only in the extreme case of riding on paved runway. Therefore, transducers rigid type or massive rigid plates are not suitable for the bicycle application. At the handlebar, we have installed a beam type piezoelectric generator which consists of a metal flexible beam upon which has been stuck a flexible piezoelectric materials (Figure 9 and 10), MFC type "Micro Fibre Composite" with a good flexibility and whose mechanical lifetime may reach several million cycles. Even with low levels of vibration, such a transducer may be deformed considerably. Let us recall that the more the piezoelectric element is deformed or bent, the more electrical energy is produced.

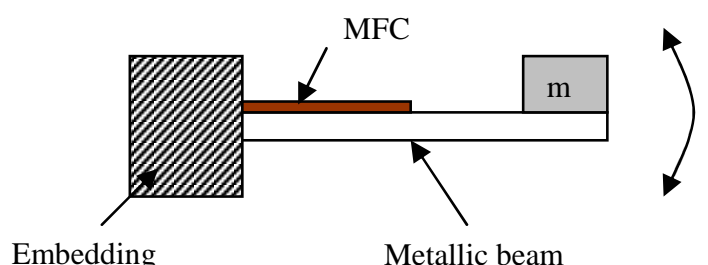

Fig. 9. Metallic beam with piezoelctric element.

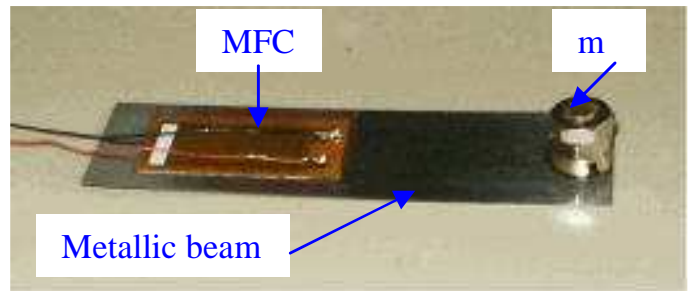

Fig. 10. Beam piezoelectric transducer using a MFC.

A proof mass $\mathrm{m}=5.32 \mathrm{~g}$ has been added at the end of the beam, to adjust its natural frequency at $12.5 \mathrm{~Hz}$, and to amplify mechanically the deformation of the piezoelectric element. The following paragraph discuss about such transducer modelling.

\section{Piezoelectric beam modelling}

The electrical behaviour of a vibrating piezoelectric transducer can be modelled. If the considered generator is excited around its resonance frequency and in the case of a little displacement for witch the movement remains linear, the structure with piezoelectric elements can be modelled by a mass + piezo + spring + damper as the one shown in figure 11 .

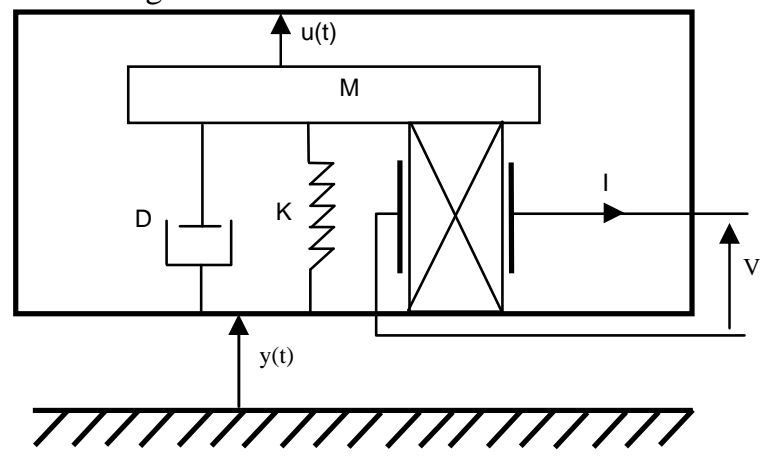

Fig. 11. Equivalent model of a vibrating piezoelectric structure
In a simplified approach, the considered structure is a rigid mass $\mathrm{M}$ bonded on a spring $\mathrm{K}$ corresponding to the stiffness of the mechanical structure, on a damper D corresponding to the mechanical losses of the structure, and on a piezoelectric disk corresponding to the bonded piezoelectric elements. The mass displacement is $\mathrm{u}, \mathrm{I}$ and $\mathrm{V}$ are the outgoing current and the voltage on the piezoelectric layer that is connected on the energy harvesting device.

The piezoelectric equations link the mechanical variable $\left(\mathrm{u}, \mathrm{F}_{\mathrm{P}}\right)$ and the electric ones $(\mathrm{I}, \mathrm{V})$ is given by the simplified set of relations (1).

$$
\left\{\begin{aligned}
F_{P} & =K_{P} u+\alpha V \\
I & =\alpha \dot{u}-C \dot{V}
\end{aligned}\right.
$$

$\mathrm{K}_{\mathrm{P}}$ is the stiffness of the piezoelectric layer when it is short-circuited; $\mathrm{C}$ is its clamped capacitance and $\alpha$ is a force factor. It can be shown that the movement $u(t)$ of the mass within the generator, for a given vibration of the generator $\mathrm{y}(\mathrm{t})$, is described by the well-known mechanical differential equation:

$$
M \ddot{u}+D \dot{u}+K u+K_{P} u+\alpha V=-M \ddot{y}
$$

The external excitation displacement $y(t)$ is considered as purely sinusoidal with a frequency closed to the resonance frequency of the structure.

For the sake of keeping this discussion simple, we consider a purely resistive load directly connected to the piezoelectric element. In this case, the voltage at the load $\mathrm{R}$ is alternative. Considering the second piezoelectric equation and the resistance load, the voltage on the piezoelectric element can be expressed in the frequency domain as a function of the displacement (5), where $\omega$ is the angular frequency.

$$
V=\frac{\alpha R}{1+j R C \omega} j \omega u
$$

Expression (6) linking voltage $\mathrm{V}$ and displacement $\mathrm{y}$ is obtained using (4) written in the frequency domain and (5).

$$
\frac{V}{y}=\frac{j M \alpha R \omega^{3}}{\left(-M \omega^{2}+j D \omega+K+K_{P}\right)(1+j R C \omega)+j \alpha^{2} R \omega}
$$

At the resonance of the structures with low viscous losses, the expression (6) can be simplified and leads to (7):

$$
\frac{V}{y}=\frac{M \alpha R \omega^{2}}{\alpha^{2} R+D+j R C D \omega}
$$

Starting from (7), the harvested power can be expressed as a function of displacement $\mathrm{y}$ and load resistance $\mathrm{R}(8)$.

$$
P=\frac{V V^{*}}{2 R}=\frac{y^{2}}{2} \frac{M^{2} \alpha^{2} R \omega^{4}}{\left(\left(\alpha^{2} R+D\right)^{2}+(R C D \omega)^{2}\right)}
$$

For weakly electromechanically coupled structure, the variable $\alpha$ is close to zero, which leads to a simplified expression of harvested power (8). In this case the harvested power reaches a maximum $\mathrm{P}_{\max }$ for an optimal load $\mathrm{R}_{\mathrm{opt}}$.

$$
P=\frac{y^{2}}{2} \frac{M^{2} \alpha^{2} R \omega^{4}}{\left(1+(R C \omega)^{2}\right) D^{2}}
$$




$$
R_{\text {opt }}=\frac{1}{C \omega} \text { and } P_{\max }=\frac{y^{2} M^{2} \omega^{2} \alpha^{2}}{4 D^{2} C}
$$

In the case of a transducer vibrating out of its own mechanical resonance, the maximum harvested power is also obtained for the same optimal load $R_{\text {opt }}$.

We can considered that the applied force at the centre of the diaphragm is $F=M y \omega^{2}$. Expression (9) can be rewritten:

$$
P=\frac{F^{2}}{2} \frac{\alpha^{2} R}{\left(1+(R C \omega)^{2}\right) D^{2}}
$$

We confronted the theoretical results to the experimental ones. To achieve this, we have measured the electrical power recovered through the transducer mentioned above. The experiment was carried out using a shaker that simulates the vibrations of the bike at $12.5 \mathrm{~Hz}$ frequency. The vibration test was purely sinusoidal which is $0.5 \mathrm{~g}$ or $5 \mathrm{~m} / \mathrm{s}-2$ amplitude.

\section{A. Theorical and experimental powers comparison}

The characterization of the transducer on a bike traveling outside is feasible but not practical because not reproducible (irregular velocity, random profile runway). This characterisation requires the testing of different load values, for example, a series of resistance and measuring for each of them the voltage produced in order to deduce the corresponding power. Therefore, we have carried out the characterization differently: inside and on test bench, see figure 12. The frequency was set $12.5 \mathrm{~Hz}$ corresponding to the frequency of the bike. The source of vibration is simulated by a shaker. The frequency and amplitude of vibration are selected to be close to that characteristic of the bike (at the frequency where there is maximum acceleration). This frequency is about $12.5 \mathrm{~Hz}$ by reference to the frequency band defined above. Without use of electronic converter, a resistive load is directly connected to the electrodes of the active material. Figure 13 tells us about the power level according to the resistive load experimental and theoretical.

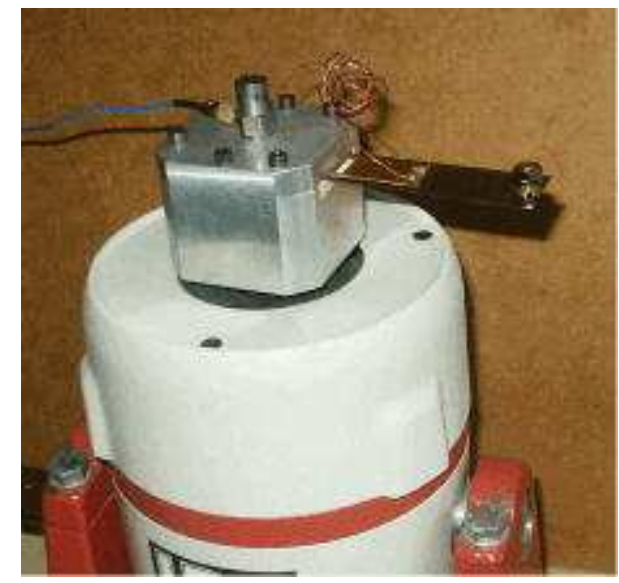

Fig. 12. Experimental set up

The power provided to the resistive load $\mathrm{R}$ is given by $\mathrm{P}=\mathrm{V}^{2}$ eff $/ \mathrm{R}$ where $\mathrm{V}_{\text {eff }}$ the RMS voltage measured between the two electrodes of piezoelectric material.
We have measured the electric power that can produce the piezoelectric generator designed. An experiment with a shaker, which simulates purely sinusoidal vibrations at $12.5 \mathrm{~Hz}$ and $0.5 \mathrm{~g}$ or $5 \mathrm{~ms}^{-2}$, has produced $3.5 \mathrm{~mW}$ power if an optimal resistance of $100 \mathrm{k} \Omega$ is connected directly to the generator output (see Figure 13). Such power is enough to power a LED lamp.

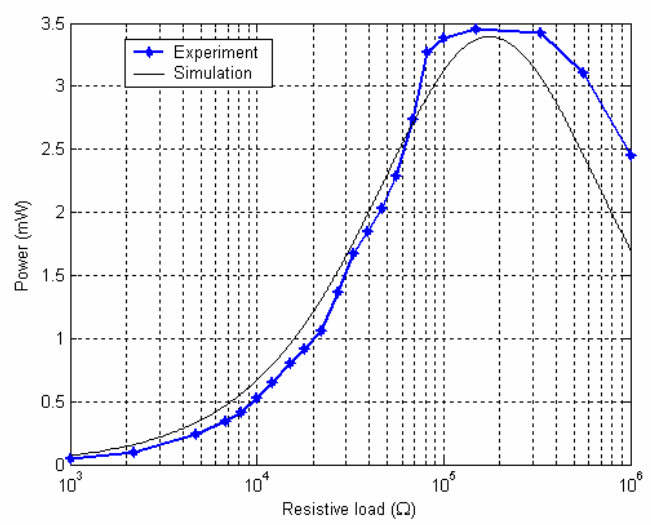

Fig. 13. Experimental and theorical powers produced versus resitive loads, acceleration $5 \mathrm{~ms}-2$ and frequency $12,5 \mathrm{~Hz}$ with $\mathrm{M}=7 \mathrm{~g}, \alpha=0.00047 \mathrm{~N} / \mathrm{V}$ and $\mathrm{D}=0.0419 \mathrm{Nm}^{-1} \mathrm{~s}^{-1}$

\section{B. Experimental results on the bike}

We then mounted on the handlebar of the bicycle experimental piezoelectric generator supplying a lamp, which consists of a high brightness LED (Figure 14) associated with an electronic circuit that permit to have either a continuous lighting or flashing lighting.

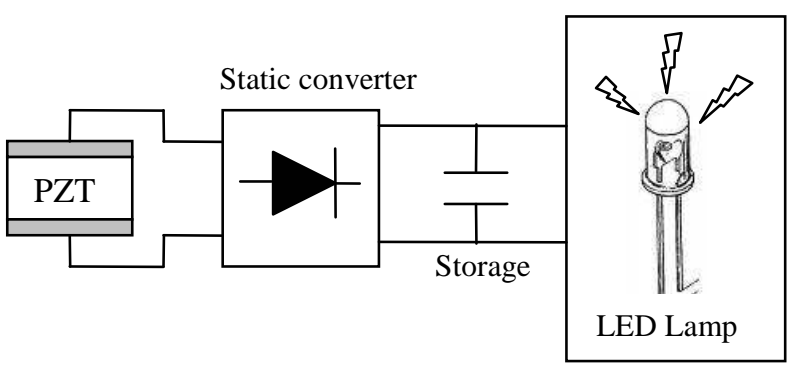

Fig. 14. Bike LED lamp supply using piezoelectric material

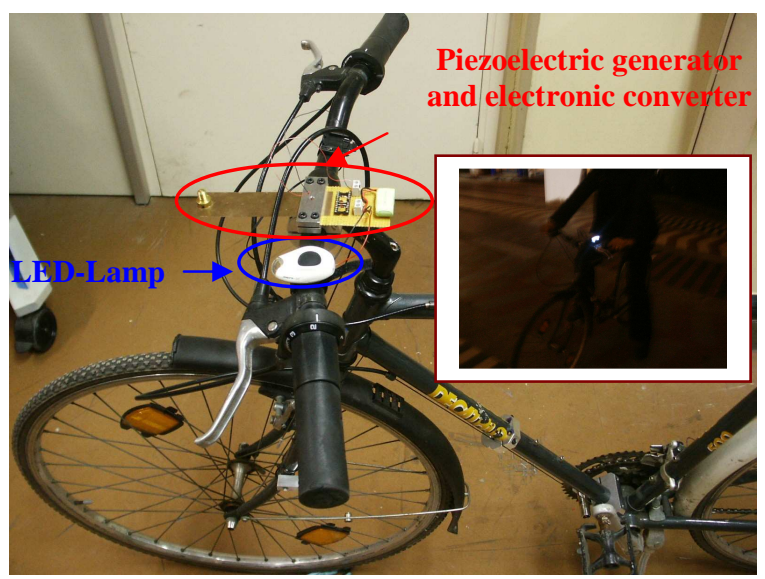

Fig. 15. Piezoelectric generator installed on the handlebar of a bicycle to power a lamp. 
Experiences reveal that on smooth track, such as a bicycle path, the light produced is lower than that obtained on uneven surface tracks, such as a paved runway. Those statements show at one side that the vibrations of the bicycle on uneven surface tracks are vastly superior to those obtained on a cycle track and at the other side that piezoelectric generator is a clean power source that could be an alternative to dynamo and improve battery lifetime.

\section{Price and efficiency of the proposed generator, compared with a dynamo}

Dynamo collects a small part of the mechanical energy provided by the cyclist to convert it into electricity. Normally, with dynamo, one never needs to worry about changing or recharging the batteries. Nevertheless, it often breaks down, or causes a rolling resistance excessive. But as everything is a matter of quality and price: it is true that the standard dynamos installed on new bike are often of poor quality, there are also some very good products [5]. There are three types of dynamo:

- The most popular type is the sidewall-running bottle dynamo, it runs on the side of the tire, costs inexpensive, starting from $€ 5$. Their major drawback is to slip under the rain, unless to take a top model, for example Dymotec S6 of Busch and Müller, which can be fitted with a wire brush roller "guaranteed" anti-slippage. Standard dynamos present low efficiency of about $20 \%$. So for a cyclist who provides on average $100 \mathrm{~W}$ power, $20 \%$ efficiency means that $15 \mathrm{~W}$ is taken from the cyclist to run the dynamo.

- The "bottom bracket" models mount just behind the crankset and run on the centre of the rear tire. Their largest area drive limit slipping and their performances are often higher. There is currently a model manufactured by Basta, 50 euros. One disadvantage: they are very vulnerable to projections of mud and water, and must therefore be strong for any challenge.

- The "hub dynamo" models are contained within the hub of the front wheel, and produce the least drag, but require change or rebuilding of the front wheel, and are the most expensive to purchase. Hub generators have some residual drag even when the lights are off, since the generator is always running. Their performance is exceptional, they never break down. By way of comparison: if in general sidewall dynamos have $20 \%$ efficiency, the same kind but more expensive can reach $40 \%$ to $70 \%$; "bottom bracket" dynamos efficiency generally range from $50 \%$ to $70 \%$, and that of hub dynamos up to $90 \%$.

Table II compares some classical dynamos to piezoelectric generator. It must be noticed that this study is a prospective, thus, the generator that we have developed exhibits poor performance and is far from a marketable prototype. It is interesting to notice today that the benefits which can offer such a generator are for instance light weight because less than $50 \mathrm{~g}$, no drag and no wear because there is no friction on the tire and the mechanical energy recovered comes from the waste, but not an extra energy dragged from the cyclist, reliable because doesn't suffer from the weather (if placed in a waterproof box). Unlike the classic sidewall dynamo that slips when there is rain or snow.

TABLE II. - Dynamos performances comparison

\begin{tabular}{|c|c|c|c|c|}
\hline Type & $\begin{array}{c}\text { Bottle } \\
\text { dynamo }\end{array}$ & $\begin{array}{c}\text { Hub } \\
\text { dynamo }\end{array}$ & $\begin{array}{c}\text { Roller } \\
\text { dynamo }\end{array}$ & Piezoelectric \\
\hline Model & $\begin{array}{c}\text { B \& M } \\
\text { Dymotec } \\
6\end{array}$ & $\begin{array}{c}\text { Shimano } \\
\text { DH- } \\
\text { 3N71 }\end{array}$ & $\begin{array}{l}\text { Union- } \\
\text { Basta } \\
8601\end{array}$ & $\begin{array}{c}\text { MFC } \\
2814 \mathrm{P} 2 \\
\text { based }\end{array}$ \\
\hline Efficiency & $40 \%$ & $53 \%$ & $<30 \%$ & $<10 \%$ \\
\hline $\begin{array}{c}\text { Price } \\
\text { approx. }\end{array}$ & $36 €$ & $60 €$ & $50 €$ & $\begin{array}{c}\text { Not } \\
\text { Assigned }\end{array}$ \\
\hline $\begin{array}{c}\text { Speed } \\
\text { average }\end{array}$ & $10 \mathrm{~km} / \mathrm{h}$ & $10 \mathrm{~km} / \mathrm{h}$ & $10 \mathrm{~km} / \mathrm{h}$ & $10 \mathrm{~km} / \mathrm{h}$ \\
\hline Voltage & $6 \mathrm{~V}$ & $6 \mathrm{~V}$ & $6 \mathrm{~V}$ & $6 \mathrm{~V}$ \\
\hline Power & $3 \mathrm{~W}$ & $3 \mathrm{~W}$ & $3 \mathrm{~W}$ & $<3.5 \mathrm{~mW}$ \\
\hline Weight & $180 \mathrm{~g}$ & $680 \mathrm{~g}$ & $215 \mathrm{~g}$ & $50 \mathrm{~g}>$ \\
\hline $\begin{array}{c}\text { Drag } \\
\text { estimated }\end{array}$ & $7.5 \mathrm{~W}$ & $5.6 \mathrm{~W}$ & $10 \mathrm{~W}<$ & $35 \mathrm{~mW}<$ \\
\hline
\end{tabular}

The last ten years have seen the birth of many new types of piezoelectric materials or transducers (MFC, PFC, LIPCA, and THUNDER), each one more performing than others. Knowing the numerous patents or publications on their evolution and their mass production consequently lower prices, it is tempting to believe that if optimized geometrically a piezoelectric generator associated with a well suited electronic, is likely able to produce the 3 Watts standard required for the lighting system of a bicycle, with all the benefits that it provides.

\section{Conclusion}

We have designed a piezoelectric generator and installed it on a bicycle handlebar; the first experiments we have conducted have shown that the few $\mathrm{mW}$ that produced the piezoelectric generator is able to power LED-lamp. Under ideal conditions such as pure sinusoidal vibrations at $5 \mathrm{~ms}^{-2}$ and $12.5 \mathrm{~Hz}$, the power harvestable measured achieved $3.5 \mathrm{~mW}$ for an optimal resistive load of $100 \mathrm{k} \Omega$, power that is sufficient to recharge a battery, or to power low consumption devices.

\section{References}

[1] U. K. Singh and R. H. Middleton, "Piezoelectric power scavenging of mechanical vibration energy", Australian Mining Technology Conference, 2-4 October (2007), pages 111-118.

[2] Roundy S., Wright P. K. and Rabaye J., "A. study of lowlevel vibrations as a power source for wireless sensor nodes", Computer Communications 26 (2003) 1131-1144.

[3] Steven R. Anton and Henry A. Sodano, A review of power harvesting using piezoelectric materials (2003-2006), Smart Materials and Structures 16 (2007) R1-R21.

[4] Y. C. Shu and I. C. Lien, "Analysis of power output for piezoelectric energy harvesting systems", Smart Materials and Structures 15 (2006), pages 1499-1512.

[5] Jan Heine and Andreas Oehler, Vintage Bicycle Press Vol. 3, No. 4, 2005 